\title{
Postpartum social interactions in families of spiny mice (Acomys cahirinus) observed in a laboratory environment
}

\author{
KATHERINE SZIJARTO, RICHARD J. COFFEE, CATHERINE BOYLE, DIANE BAILEY, \\ MARISA MULÉ, DONAN IACOVONE, and RICHARD DENI \\ Rider College, Lawrenceville, New Jersey
}

\begin{abstract}
Eight mated pairs of adult spiny mice (eight males, eight females) with their litters were observed from the day before birth through Day 10 postpartum. Parent-offspring interactions in the form of the amount of time each parent spent in physical contact with its offspring were recorded. Parental interactions were also recorded by measuring (1) the amount of time the dam and sire were in physical contact, (2) their activity levels, and (3) the percentage of times the dam led and followed the sire through the home environment. Each mated pair reared its litter inside a four-compartment habitat enclosure. Observations took place on the day before birth (Day 0) and on Days 1, 5, and 10 postpartum, were recorded on videotape, and were later coded in 1-h samples using a TRS-80 Model 100 portable computer. The primary findings were that (1) adult activity levels peaked on Day 1, (2) sires accumulated significantly more contact time with offspring than did dams, and (3) dams tended to follow sires during movement sequences significantly more often than they led sires. Limited developmental trends were observed in parental and parent-offspring interactions as a function of the postpartum age of the litter. These data further define the parental role of sires in Acomys and document their contribution to social cohesiveness and mutual attachment among family members.
\end{abstract}

A growing number of studies of parental behavior in rodents have focused on the role of the sire and his direct and indirect contributions to the development of his offspring. Direct contributions are those that come from specific forms of parent-offspring interaction, and are by far more numerous in documentation of the sire's influence. Indirect contributions, those that appear to result from the sire's influence on the dam, have also been reported, although less frequently.

In studies reporting direct contributions by the sire, his presence was often the independent variable manipulated. Presence, however, has taken on the dual meaning of being either physical or "chemical," with the advancement of knowledge of the role of odor cues as determinants of behavior. A good example of this approach is the series of experiments by Csermely $(1980,1981)$, who reported that newborn mouse pups (Mus musculus) were made less active in or near the nest by the actual presence of a sire. Furthermore, the urine of the sire produced a similar effect. However, because the urine of unbred strange males also subdued activity in pups, the chemical cue hypothesis appears to be nonspecific to paternal contributions per se.

The sire's direct effect on individual behavior patterns has also been studied. For example, Mugford and Nowell

We thank Burnell Hartzell for his work in maintaining the research animals used in this study, and Donna MacKerell for assistance in preparation of the manuscript. Please address correspondence to the authors in care of: Department of Psychology, Rider College, Lawrenceville, NJ 08648.
(1972) showed that the presence of the sire during the postpartum period contributed to the development of fighting behavior in albino mouse pups. And as might be expected, others have reported that direct contact with the sire is not necessary to facilitate the development of fighting (Wuensch \& Cooper, 1981); rather, access to the sire's odor alone is sufficient.

Also numerous among these reports are observational studies of entire family units (dam, sire, and litter). These have often indicated that both parents participate in the caretaking of their young. Preistnall and Young (1978), for example, reported that Mus sires did not differ from dams in the amount of nest building they performed or in the frequency of licking or retrieving pups. This pattern of paternal behavior has been observed in several other rodent species (Hartung \& Dewsbury, 1979). Elwood (1975) characterized the sire's contribution to caretaking in Mongolian gerbils (Meriones unguiculatus) as one of providing high levels of tactile stimulation of the young. The sire's role in family interactions involved behaviors such as licking the pups and walking and lying on the pups.

In reviewing such data, it becomes difficult to separate direct from indirect paternal contributions. For example, Elwood and Broom (1978) postulated that the paternal contribution in gerbils serves to increase the temperature at the nest site, which facilitates the rate of development of the pups. Pups reared in family units with their sire developed to the point of eye opening faster than pups reared without the sire. Likewise, a facilitative, but ap- 
parently indirect, effect of males was reported by Fullerton and Cowley (1971), who showed that chemical cues from males influenced the behavior, growth, and development of young mice (Mus). They found that pups exposed to the odor of adult males gained weight, developed more rapidly, were heavier when weaned, and were more active in open-field tests than those exposed to the odor of a group of adult females.

The present observational study of families of spiny mice (Acomys cahirinus) was a longitudinal analysis of parental and parent-offspring interactions. Spiny mouse pups are precocial, born covered with hair and with their eyes open. They show intact motor and sensory capabilities immediately following birth. They are unique subjects for testing hypotheses generated by what is already known about paternal behavior in rodents. One question of particular interest here was whether spiny mouse sires contributed to parent-offspring interaction and whether there was a developmental factor in this contribution. For example, there would seem to be little demand for the sire's presence or proximity as a temperature source by precocial offspring born with hair. Another concern of these observations was to determine any pattern of change in the parental relationship as the pups developed.

Measures of individual behavior by parents included their activity and locomotion in a family habitat cage. Measures of their social interaction included the duration of physical contact and the degree to which the dam led or followed the sire during coordinated movement. Measures of parent-offspring interaction included the duration of physical contact with pups.

\section{METHOD}

\section{Subjects}

The subjects were eight mated pairs of adult spiny mice (Acomys cahirinus), eight males and eight females, and their litters. All adults had had prior breeding experience. The litters were born during the period of the study and ranged from 1 to 4 pups. During the period of the study, the subjects were maintained under continuous light and were kept separate from the main colony.

\begin{abstract}
Apparatus
Each dam, sire, and litter were housed together in a habitat enclosure that comprised four clear plastic compartments interconnected by two networks of plastic tunnels. This apparatus was installed in a small test room apart from the main colony. The resulting configuration represented six movement zones. The floor of each compartment was covered with a layer of wood shavings, and two of the compartments contained both water and Purina Rat Chow. The bedding was not changed during the observation period. The test enclosure was washed thoroughly with an Alconox solution prior to the introduction of a family. Videotape apparatus was used to record all observations. A TRS-80 Model 100 portable computer was used to code the videotapes. The resulting records of the observation sessions consisted of streams of behavioral codes matched to the elapsed clock time at which the code was entered (Deni, Szijarto, Eisler, \& Fantauzzo, 1984).
\end{abstract}

\section{Procedure}

Four 60-min videotapes were recorded over the course of litter development. Separate recordings were obtained between 1400 and $1700 \mathrm{~h}$ on Day 0 (the day before birth) and Days 1,5 , and 10 postpartum. The subjects were not physically disturbed during the course of the study.
The videotapes were coded using two separate passes. The first coding was to measure the duration (in seconds) of three categories of physical contact: (1) dam touching pup(s), (2) sire touching pup(s), and (3) dam touching sire. Each category was represented by a pair of keys on the portable computer used as the recording device. Each pair consisted of a "start"' key, pressed when contact was initiated, and a "stop"' key to end timing. The second coding series was to measure locomotion by the dam and sire. The frequency of movement from zone to zone within the apparatus was recorded. In addition, a sequential analysis of the tendency of the dam to lead or follow the sire was done. An interrater reliability check on each coding series showed that agreement on all measures was above $90 \%$.

\section{RESULTS}

\section{Duration of Contact with Pups}

The raw data on the duration for each of the three categories of physical contact at each postpartum age level were converted to minutes, and two analyses were done.

A two-factor mixed analysis of variance (Kirk, 1968, p. 248) was done to test for differences as a function of sex of parent (the between-subjects factor) and postpartum age of litter (the within-subjects factor). There was a significant main effect of sex of parent $[\mathrm{F}(1,14)=30.3$, $\mathrm{p}<.01$ ]. Sires spent much more time in physical contact with their pups (mean $=39.9 \mathrm{~min}, \mathrm{SD}=60.1$ ) than did dams (mean $=12 \mathrm{~min}, \mathrm{SD}=12.0$ ). The developmental trends in this measure, although not statistically significant, indicated parental sex differences. Table 1 shows that sires generally increased their physical contact with pups as they matured, whereas dams maintained a steady level on this measure.

The effect of the postpartum age of litters on the physical contact between dams and sires was also tested. A within-subjects analysis of variance was done on the parental contact scores from each age level, but the age factor was not statistically significant. The developmental trend, however, was smooth and showed a drop in duration of contact at the day of birth, followed by a gradual return to the prepartum level. The means in minutes for contact between dams and sires at Days 0 (before birth), 1,5 , and 10 were $14.0,7.3,10.2$, and 14.4 , respectively (SDs were 20.5, 11.0, 19.4, and 21.6, respectively).

\section{Activity Level of Parents}

The raw data on activity level were derived from counting the number of times each adult moved from zone to

Table 1

Means and Standard Deviations of Duration (in Minutes) of Physical Contact with Pups by Dams and Sires at Three Postpartum Ages

\begin{tabular}{ccrc}
\hline Sex of Parent & Postpartum Age & Mean & SD \\
\hline \multirow{4}{*}{ Dam } & Day 1 & 14.0 & 20.3 \\
& Day 5 & 8.3 & 17.5 \\
& Day 10 & 13.8 & 16.6 \\
& Day 1 & 29.0 & 22.1 \\
Sire & Day 5 & 44.3 & 18.9 \\
& Day 10 & 46.4 & 18.5 \\
\hline
\end{tabular}


Table 2

Means and Standard Deviations of Percentage of Times Dams Led and Followed Sires During Sequences of Movement Through the Six Zones of the Test Habitat the Three Postpartum Ages

\begin{tabular}{llrr}
\hline & Movement & & \\
Postpartum Age & Pattern & Mean & \multicolumn{1}{c}{ SD } \\
\hline \multirow{2}{*}{ Day 0 (before birth) } & Lead & 0.7 & 1.4 \\
& Follow & 20.4 & 30.3 \\
Day 1 & Lead & 20.2 & 29.6 \\
& Follow & 12.6 & 22.8 \\
Day 5 & Lead & 4.5 & 4.8 \\
& Follow & 18.1 & 26.6 \\
Day 10 & Lead & 0.0 & 0.0 \\
& Follow & 25.1 & 26.6 \\
\hline
\end{tabular}

zone within the test enclosure. Another two-factor mixed analysis of variance was done to test for the effects of sex of parent and postpartum age of litter. There was a significant postpartum age effect $[\mathrm{F}(3,42)=9.19$, $\mathrm{p}<.001]$. Overall locomotor activity peaked on Day 1 . Post hoc Newman-Keul's tests showed Day 1 to differ from all other levels. The means for Days $0,1,5$, and 10 , respectively, were $36.4,170.8,21.8$, and 21.3 (SDs were $66.9,172.8,15.7$, and 13.6 , respectively).

\section{Patterns of Social Movement by Parents}

The raw data on social movement were in the form of the number of sequences of the dam leading or following the sire from zone to zone. These scores were corrected for differences in overall activity levels by transformation into percentages. The resulting percentages indicated (1) the proportion of zone moves by the dam in which she led the sire and (2) the proportion of moves in which she followed the sire.

These scores were tested with a two-factor withinsubjects analysis of variance (Kirk, 1968, p. 237) with both postpartum age $(0,1,5$, and 10 days) and movement pattern (lead, follow) as within-subjects factors. There was a significant difference in the percentage of time the dam led versus followed the sire $[F(1,7)=7.39$, $\mathrm{p}<.05]$. Dams followed the sire three times more often than they led. The percentage means were 19.0 and 6.4, (SDs were 25.8, and 16.5), respectively.

Although not statistically significant, the developmental trends in these social movement scores indicated that a stable developmental pattern was broken on Day 1 postpartum. Table 2 shows Day 1 to be unique in that on that day dams led sires more than they followed them.

\section{DISCUSSION}

The important findings of this observational study of spiny mice concerned the role of the sire in parental and parent-offspring interactions. Sires showed considerable attachment behavior toward their offspring, in that they exceeded dams in the level of physical contact with pups. This observation represents an obvious direct contribution to the process of parent-offspring attachment. Sires also showed a developmental progression in the maintenance of physical contact with their pups, in that they displayed more huddling as the pups matured. Dams did not show this progression, but maintained a steady lower level.
An indirect contribution was also noted in that dams followed sires around the habitat enclosure more frequently than they led them. Sires attracted dams during periods of social movement, and this behavior can also be seen as contributing to the maintenance of a cohesive social unit. The single exception to this finding occurred on Day 1 postpartum when the data were reversed: Dams led sires more than they followed. In these Day 1 samples, sires were observed to engage in vigorous copulatory behavior with dams. When dams followed sires, however, the behavior included neither sexual nor aggressive interactions.

These processes in spiny mice can be compared with those found to be operating in studies of family interaction in altricial mouse species. In altricial species, the pups' need to conserve body heat has been identified as a controlling factor in the high incidence of paternal tactile stimulation (Dudley, 1974; Elwood \& Broom, 1978). Since spiny mice are born with hair and locomotor capacity (two factors that contribute to independent regulation of body temperature), it would seem reasonable not to expect sires to engage in high levels of huddling with their pups. This was not the case, however, and the result may call for an explanation based on a more complex conception of family attachment processes.

As precocial offspring, spiny mouse pups pose more of a problem for a parent seeking to maintain proximity and to monitor the pup's position in a complex environment. It may be that the present data indicate a specific paternal role in caregiving in that sires frequently huddle with their pups (a direct contribution), and when sires move, they are likely to elicit following responses by the dam (an indirect contribution). The effect in any case is likely to be one of increased family cohesiveness as dams follow sires and sires keep in regular huddling contact with offspring.

\section{REFERENCES}

Csermely, D. (1980). Locomotor activity in newborn mice: I. Effect of paternal presence. Bollettino di Zoologia Agraria E di Bachicoltura, 47, 139-142.

CSermely, D. (1981). Locomotor activity in newborn mice: II. Presence and influence of a male pheromone. Bollettino di Zoologia Agraria E di Bachicoltura, 48, 179-182.

Deni, R., Szijarto, K., Eisler, A., \& Fantauzzo, C. (1984). BaSiC programs for observational research using the TRS-80 Model 100 portable and Model 4 computers. Behavior Research Methods \& Instrumentation, 15, 616.

Dudley, D. (1974). Paternal behavior in the California mouse Peromyscus californicus. Behavioral Biology, 11, 247-252.

Elwood, R. W. (1975). Paternal and maternal behaviour of the Mongolian gerbil. Animal Behaviour, 23, 766-772.

Elwood, R. W., \& Broom, D. M. (1978). The influence of litter size and parental behaviour on the development of Mongolian gerbil pups. Animal Behaviour, 26, 438-454.

Fullerton, C., \& Cowley, J. J. (1971). The differential effect of the presence of adult male and female mice on the growth and development of the young. Journal of Genetic Psychology, 119, 89-98.

Hartung, T. G., \& Dewsbury, D. A. (1979). Paternal behavior in six species of muroid rodents. Behavioral and Neural Biology, 26, 466-478.

KIRK, R. E. (1968). Experimental design: Procedures for the behavioral sciences. Belmont, CA: Brooks/Cole.

Mugford, R. A., \& Nowell, N. W. (1972). Paternal stimulation during infancy: Effects upon aggression and open-field performance of mice. Journal of Comparative and Physiological Psychology, 79, 30-36.

Priestnall, R., \& Young, S. (1978). An observational study of caretaking behavior of male and female mice housed together. Developmental Psychobiology, 11, 23-30.

Wuensch, K., \& CoOPer, A. (1981). Preweaning paternal presence and later aggressiveness in male Mus musculus. Behavioral and Neural Biology, 32, 510-515.

(Manuscript received for publication January 16, 1985.) 